\title{
Applications of Focused Ion Beam (FIB) on Yeast Cell \& SARS Virus
}

H. L. Hing ${ }^{1}$, C. Burkhardt ${ }^{2}$, P. Gnauck ${ }^{2}$, S. Sally ${ }^{3}$,

H. Gelderbloms ${ }^{4}$, Y. Muranaka ${ }^{5}$

M.A. Kaswandi ${ }^{1}$, A.H. A. Aziz1 \& A.Z. Sahalan ${ }^{1}$

${ }^{1}$ Faculty of Allied Health Sciences, Universiti Kebangsaan Malaysia

(National University of Malaysia) Kuala Lumpur

${ }^{2}$ Natural \& Medical Science Institute (NMI) Germany, Reutlingen, Germany

Electron Microscope Unit, Australia National University, Canberra, Australia.

${ }^{4}$ Robert Koch Institut, Berlin, Germany

${ }^{5}$ Research Equipment Center, Hamamatsu University School of Medicine, Hamamatsu, Japan

The focused ion beam (FIB) is a relatively novel technique to biomedical electron microscopy as it open up new means for the observations and examinations of a wide range of biomedical and biological materials (1). The focused ion beam, or FIB tool has been utilized mainly in the fields of material sciences and industry. The (FIB) uses high-energy gallium ions to precisely and accurately section or mill samples. Lately FIB method have been used to prepare biological samples such as yeast cells [2,3] and virus particles. Yeast cells Schwanniomyces occidentalis $S$. occidentalis were prepared by vacuum sucking them into cellulose tubing, plunge freezing them in liquid nitrogen, followed by chemical fixation in glutaraldehye and postfixed with osmium tetroxide, dehydrated in a series of ascending alcohol concentration up to absolute alcohol, then freeze dried overnight. In the case of SARS virus, the tissue culture containing virus particles was chemically fixed with glutaraldehyde, dehydrated in ascending order of alcohol concentrations and then freeze dried. Yeast cell and virus samples were then mounted on stubs and put into the JEOL Oryza FIB and/or a Zeiss Cross-Beam for FIB milling

The FIB sectioning proceeded in a step-by-step fashion to reveal internal structures of interest for Scanning Electron Microscopy observation. All the samples were milled both longitudinally and in cross-section. The FIB method allowed a single yeast cell to be sectioned at any desired location irrespective of its place and location, whereas the virus particles were too fragile for targeted analysis and were simply milled. The samples were prior coated

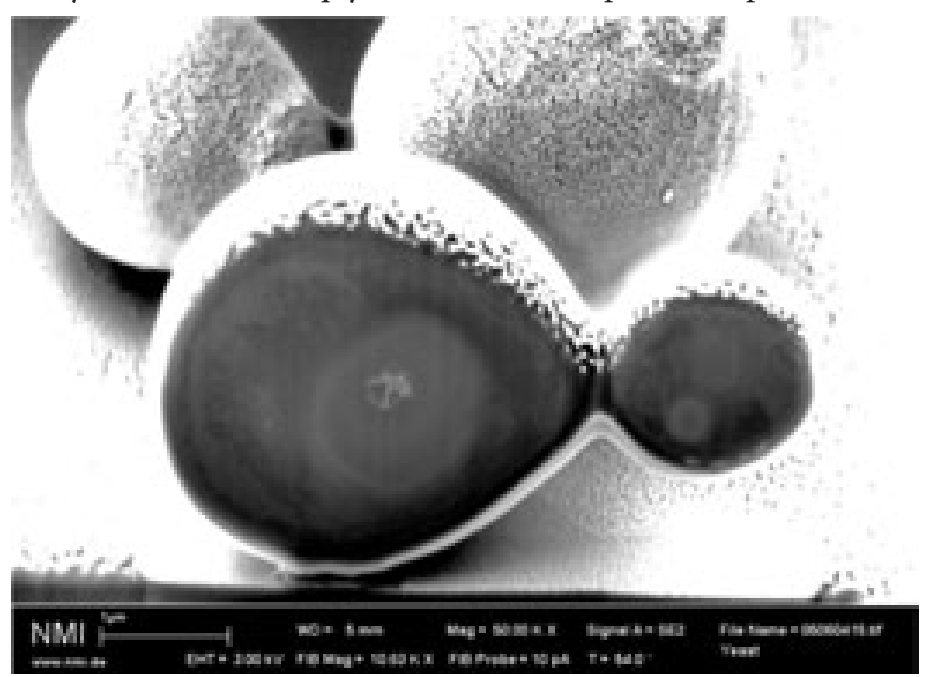

Fig 1: FIB Milled yeast cell $(p I=2 p a)$.

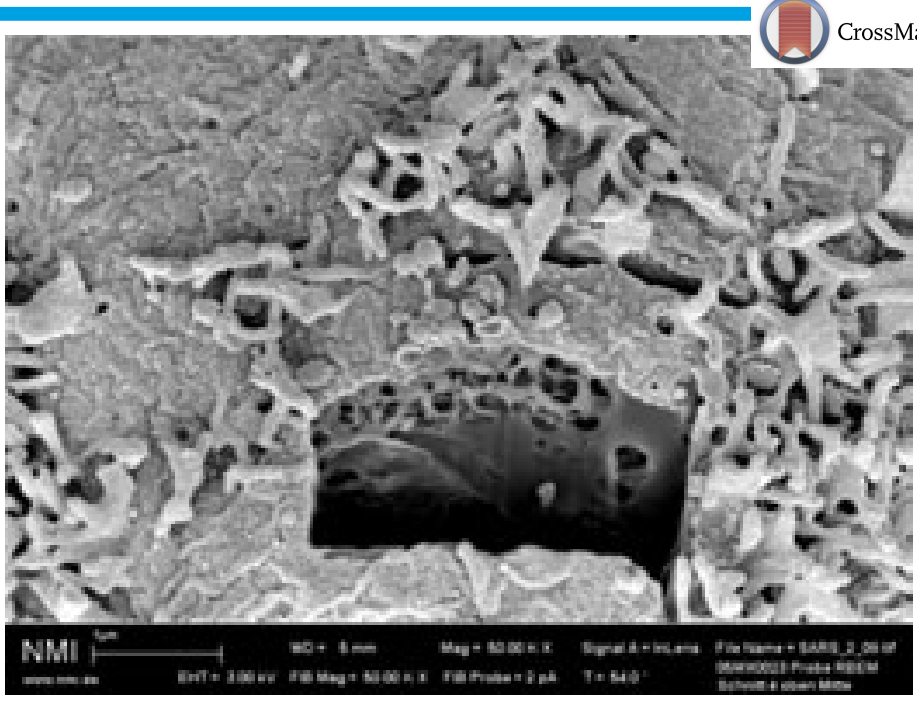

Fig 2: SARS virus milled at $2 p A$.

with a 20nm layer of gold to strengthen their outer layers during the milling process. The milling current was decreased to $2 \mathrm{pA}$ from 10 pA and $1.8 \mathrm{nA}$ (Fig 1). The lower the probe current for milling, the less damage to the internal structure of the yeast and tissue culture (Fig 2, 3). A higher current will destroy the internal structure and damages the external structure. Enhancement of FIBed specimens for SEM observations may be done by coating the prepared samples with osmium vapor, or thin films of chromium or gold. The FIB method allows easy target selection of cell specific sites for characterization of internal structures in biological systems.

\section{Results \& Discussions}

Observation of the FIB-milled structures showed that the killer yeast cell needed proper fixation for the enhancement of its internal structure and further coating with gold to enhance the strength of the cells so that it could withstand the current probe of the gallium ion beam. After initial high-current milling to rough-locate the area of interest, a low current probe was then utilized in order to not damage the surrounding area of the FIB-milled samples. The low probe current is also critical to preserve the delicate internal structure of the yeast cell: the vacuole, fat globules, and crystals in the vacuole. The dual beam FIB was very useful in that it also allowed the intermittent switching from the milling mode to scanning mode before proceeding to the next stage of milling. This is a great help in precisely locating the structures of interest and to

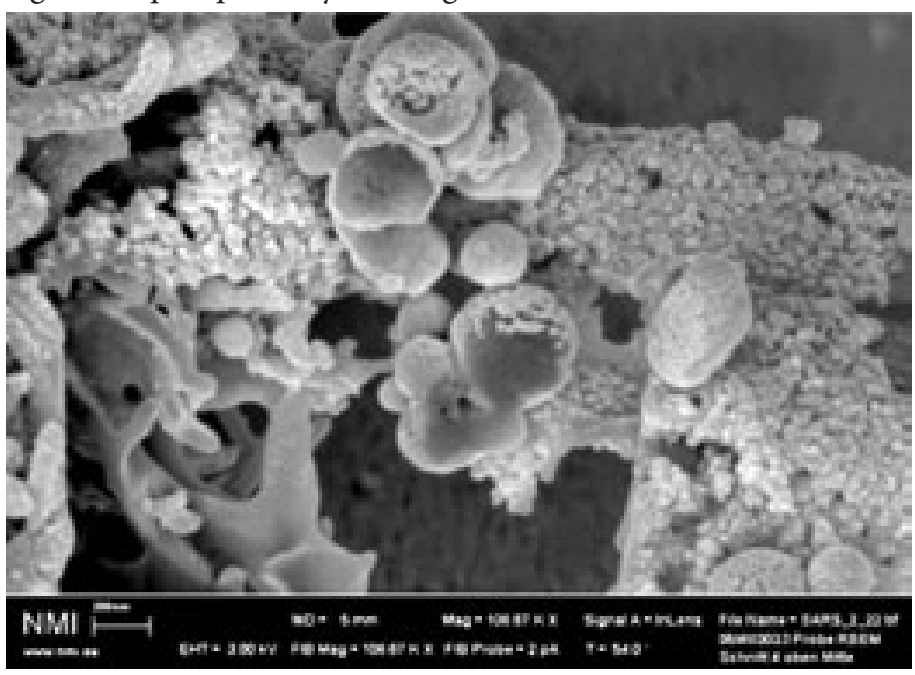

Fig 3: Yeast cell milled at $1.8 n A, 10 p A$ to $2 p A$ 
minimize structural damage to the specimen. The probe current value is also very crucial in preparing good FIB-milled samples for further observation as seen from the reduction in probe current from the initial stage ( $1.8 \mathrm{nA}$ on C. albicans) to $10 \mathrm{pAon} S$. occidentalis to $2 \mathrm{pA}$ (Fig 1). The SARS virus manifested an oblong particle shape, which differed from the vesicles/blebs on the tissue cell. Although it is possible to mill the virus particle, the protein core was not seen (Fig 2,3). Therefore, we propose that in order to mill the virus particle, it would be advisable to use pure virus particles only to eliminate the possibility of milling the blebs/vesicles instead of the virus itself.

\section{Conclusions}

The low probe current of the focused ion beam (FIB) system has allowed the internal structure of the biomedical and biological materials to be seen and the revealed structures are clearly comparable to that of a freeze-fracture section. Further research using cryo-FIB for biological/biomedical materials is deemed necessary as it would allow the samples to be in their native state when examined. Although milling of virus particles does not show any favorable images at this time, the ability to mill very small particles was demonstrated.

\section{Acknowledgements}

The authors would like to thank Dr. Sally Stowe, EM Unit, ANU, Canberra, Australia, Dr. Peter Gnauck of Zeiss, Germany \& Ms Normalawati Shamsudin for providing FIB instruments and valuable assistances. This work was presented at the Microscopy and Microanalysis meeting in Fort lauderdale, FL, August 2007, Reference Microsc Microanal 13 (Suppl 2) 2007, pp 1528-29.

\section{References:}

[1]. Monroe, P.R. \& Dickson, M.A. 2001. Proc. of the 10th Scientific Conference Electron Microscopy Soc of Malaysia, Kuala Lumpur, Malaysia (2001): 8.

[2]. H.L. Hing, Y. Muranaka, S. Normalawati, M.A. Kaswandi, A.H. Abd. Aziz, A.Z. Sahalan, Aoshima \& Y. Tsutsui. J. Electron Microscopy Soc Thailand

(2004) 17: 5.

[3]. H. L. Hing, C. Burkhardt, P. Gnauck, S. Sally, H. Gelderbloms, Y. Muranaka, M.A. Kaswandi, A.Z. Sahalan \& M.S. M Yasin. J. Electron Microscopy Soc. Thailand (2006) 19: 4

[4]. The contributions Dr. Sally Stowe, EM Unit, ANU, Canberra, Australia \& Dr. Peter Gnauck of Zeiss, Germany, Ms Normalawati Shamsudin is greatly acknowledged.

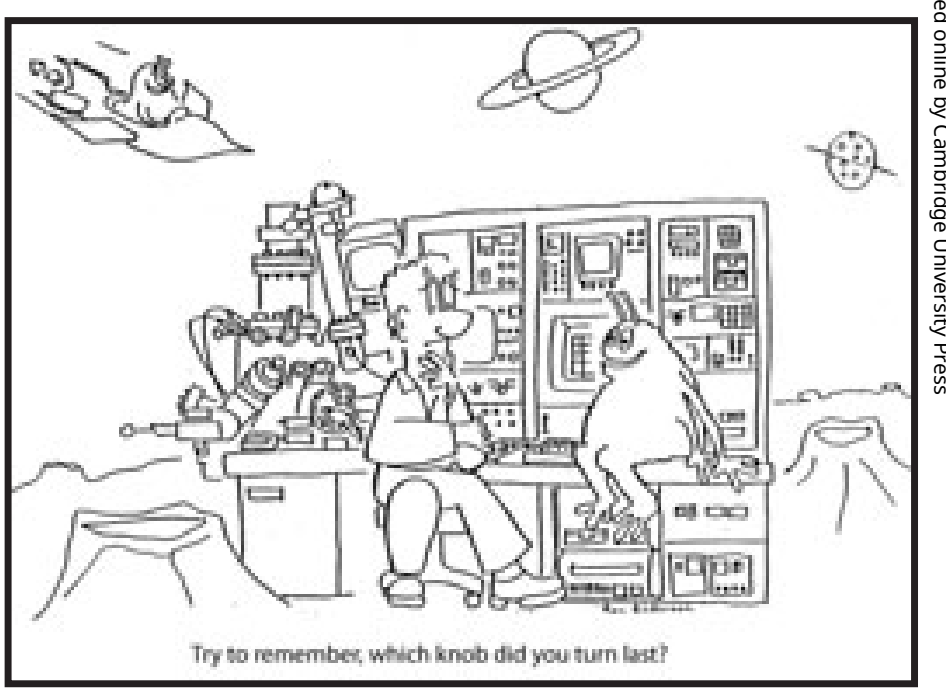

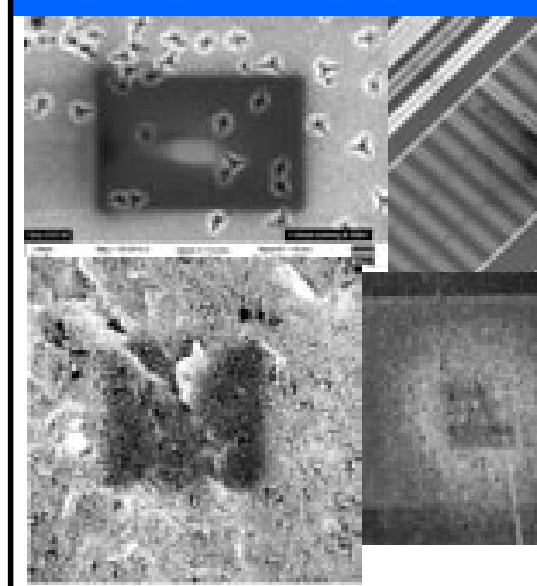
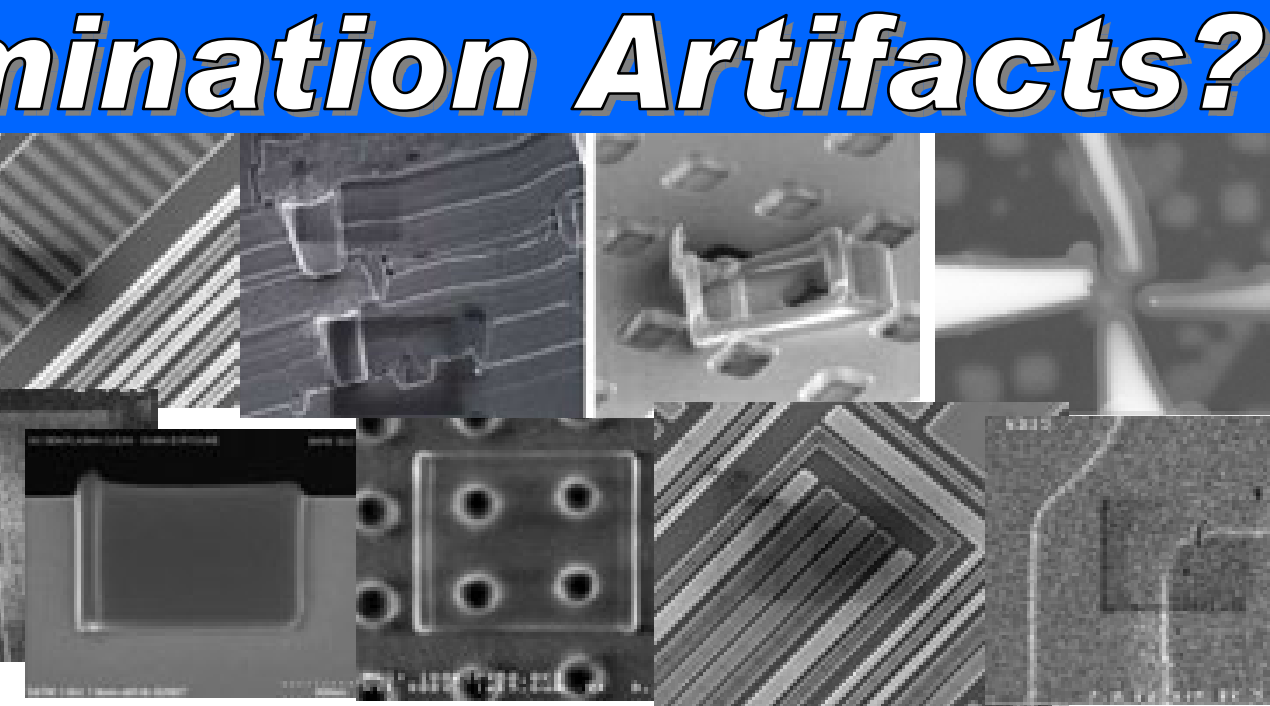

\section{The Remedy: Evagtron ${ }^{\circledR}$ Cleaning}
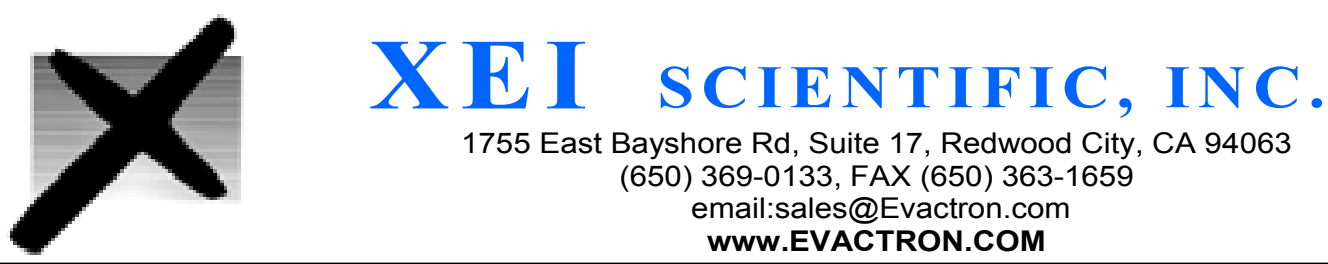

1755 East Bayshore Rd, Suite 17, Redwood City, CA 94063

(650) 369-0133, FAX (650) 363-1659

email:sales@Evactron.com

www.EVACTRON.COM 